\title{
Modulation of Neurotransmission by a Specified Oregano Extract Alters Brain Electrical Potentials Indicative of Antidepressant-Like and Neuroprotective Activities ${ }^{*}$
}

\author{
M. Hasan Mohajeri ${ }^{1 \#}$, Regina Goralczyk ${ }^{1}$, Wilfried Dimpfel ${ }^{2}$ \\ ${ }^{1}$ DSM Nutritional Products Ltd., R \& D Human Nutrition and Health, Basel, Switzerland; ${ }^{2}$ NeuroCode AG, Wetzlar, Germany. \\ Email: "hasan.mohajeri@dsm.com
}

Received December $10^{\text {th }}, 2011$; revised January $19^{\text {th }}, 2012$; accepted February $15^{\text {th }}, 2012$

\begin{abstract}
Different behavioral states are characterized by distinct patterns of global brain activity. Therefore, the biological effects of herbal extracts on brain functions can be assessed by analyzing the local field potentials, the so-called electropharmacogram analysis. Inspired by our recent findings that a specified oregano extract $(\mathrm{OE})$ exhibited a triple-reuptake activity in vitro, this extract was tested in model of Tele-Stereo-electroencephalogram (EEG) to elucidate how OE affects the electrical brain activity in freely moving rats. Furthermore, discriminant analysis was performed to compare the electric brain activity of four standardized brain regions with those produced by several reference compounds, representing a whole variety of clinical indications. Oral intake of $\mathrm{OE}$ produced fast and robust dose and time dependent EEG alterations consisting of significant changes of spectral power in comparison to controls. Strongest effects were seen with respect to alpha1, alpha2 and beta1 waves representing an activation of serotonergic, dopaminergic and glutamatergic neurotransmission, respectively. Moreover, the discriminant analysis revealed that OE's pattern of activity locates in close vicinity to antidepressant and neuroprotective compound. The presented data support the hypothesis suggesting the use of $\mathrm{OE}$ as a neuroprotective dietary supplement to promote mood, motivation and mental wellbeing.
\end{abstract}

Keywords: EEG; Dopamine; Serotonin; Noradrenalin; oréVida; Diet; Mood; Therapy

\section{Introduction}

Coping with the psychosocial challenges of modern lifestyle may involve a stress response, which can serve an adaptive function to the situation. Even though beneficial in normal circumstances, repetitive continuous stress is maladaptive leading to mood disturbances, and in excessive cases, or in individuals with genetic predispositions, to psychiatric disorders [1]. A reduction of serotonin and dopamine levels in the brain affects mood and mental wellbeing evidenced by waning motivation and increased anxiety $[2,3]$. Nutritional compounds may be valuable to improve mood and motivation in humans without the risk of side effects [4]. They exert their cellular action by interacting with biochemically defined targets on the cell membranes modulating the functions of receptors, enzymes, ion channels, and transporters. The interaction of constituents of nutritional compounds with cell-surface recaptors, such as neurotransmitter receptors, induces a signaling cascade resulting in alteration of the conductance

\footnotetext{
*The authors declare that they have no competing financial interests.

${ }^{\#}$ Corresponding author.
}

of involved ion channels in the central nervous system (CNS) $[5,6]$. The communication between neurons is governed by such alterations, because the electric activity of neurons depends on the set of momentarily active ion channels. Field potentials can be measured in different brain areas in animal models [7] and humans [6,8,9] and contain the information of larger local networks of electrically active neurons. Therefore, field potentials are believed to reflect the interaction of the chemical compounds with their targets within the concert of neurotransmission in mammalian brain. Furthermore, a coordinated balanced neurotransmitter activity within a specific brain structure may influence the behavioral outcome. Thus, the electrical changes recorded from brain areas reflect the net effect leading to the observed behavior.

Frequency analysis of the field potentials in the presence of test substance leads to the so-called electropharmacogram, which has been widely used to characterize drug actions on rat $[10]$ and human brains $[6,8]$. Electropharmacogram recordings in rats may reveal information regarding neurotransmitter activity and a possible clinical indication in humans $[11,12]$. 
The relationship between delta waves and cholinergic transmission has been demonstrated by showing that changes in cholinergic transmission by drugs like scopolamine, metanicotine, biperiden and acetylcholinesterase inhibitors were reflected mainly in spectral delta power [13]. Moreover, theta waves have been recognized as being influenced by drugs acting at the noradrenalin alpha2 receptor and pre-synaptic interaction with this receptor leads to drowsiness and sleep and changes of theta waves [14]. Additionally, alpha2 waves have been shown to be altered by drugs acting at dopaminergic receptors $[15,16]$. Thus, dopaminergic transmission could be related to spectral alpha2 frequencies [15]. Thus, Tele-Stereo-EEG technique and recorded electropharmacograms provide detailed insight into the time and dose dependency of the effects of pharmacological compounds. One of the biggest advantage of EEG, apart from being a predictive method for determining neurotransmitter function in the brain, is its high temporal resolution in the range of milliseconds [17-19]. Meanwhile, electropharmacogram data are available from rats after treatment with more than 150 compounds from a wide range of drug categories (for example, analgesics, antidepressants, neuroleptics, stimulants, tranquilizers, sedatives and narcotics), providing a valuable tool for prediction of the properties of an unknown ingredient. In general, electropharmacograms show prominent differences for drugs prescribed for different indications and are similar for drugs with similar clinical indications [10]. In addition, the discriminant analysis provides the statistical fundament for classification of single drug's effects into disease specific clusters [20-22]. All compounds within each cluster exhibit a similar pattern of changes providing a disease specific "fingerprint" of electrical activity. As an example, great similarity is shown for donepezil and galanthamine, drugs used to treat Alzheimer patients, whose well-known main mechanism of action is inhibition of acetylcholinesterase. Moreover, imipramine and paroxetine, two drugs with antidepressant action are also located in relatively close vicinity to each other. We show here that oral application of OE, a constituent of the Mediterranean diet (please see [4] for details) leads to biological effects mainly originating from the transient activation of dopaminergic, serotonergic and glutamatergic neurotransmission and provide the evidence that $\mathrm{OE}$ has neuroprotective and antidepressant-like moodimproving properties.

\section{Methods}

\subsection{Specified Oregano Extract}

The preparation of OE (oréVida ${ }^{\mathrm{TM}}$ ) by a specified twostep supercritical $\mathrm{CO}_{2}$ extraction was described elsewhere [4]. In brief, dried leaves of oregano were mechanically milled and extracted twice with carbon dioxide under super critical conditions and the specifications were analyzed by gas chromatography/mass spectrometry [4]. The major constituents of OE are carvacrol (CAS no. 499-75-2) and thymoquinone (CAS no: 490-91-5) as reported previously [4].

\subsection{Animals}

Eight adult Fisher 344 rats $(n=8$, weight about 400 g, 8 month of age, day-night converted, provided by Charles River Laboratories, 97633 Sulzfeld, Germany) were allowed to acclimatize for at least 4 weeks before the start of the study. Temperature and humidity were controlled automatically and kept at $22^{\circ} \mathrm{C} \pm 2^{\circ} \mathrm{C}$ and $44 \% \pm 5 \%$ respectively. Light hours were $18 \mathrm{~h}$ in the evening until $6 \mathrm{~h}$ in the morning. Cages, bedding, and water bottles were changed regularly, i.e. every 2 - 3 days. Standard diet (Nohrlin H10, Altromin, 32791 Lage, Germany) and water were available to the animals ad libidum.

The experiments were subjected to approvals by the local authorities responsible for animal care according to German Health Guidelines and all possible steps were taken to avoid animal suffering at each stage of the experiment. Animals were implanted with electrodes into the brain and were given two weeks for recovery from surgery (for details see the surgery section), after which the transmitter was plugged in for adaptation and experiments. During the recording rats were not restrained and could freely move but did not have access to food to avoid chewing artifacts.

EEG signals were recorded from frontal cortex, hippocampus, striatum and midbrain reticular formation of freely moving rats from inside a totally copper shielded room. Signals were wirelessly transmitted by a radio-telemetric system (Rhema Labortechnik, Hofheim, Germany, using 40 Megahertz as carrier frequency) and were amplified and processed as described earlier to give power spectra of $0.25 \mathrm{~Hz}$ resolution [20,23-25]. In short, after automatic artifact rejection signals were collected in sweeps of 4 second duration and Fast Fourier Transformed using a Hanning window. Sampling frequency was $512 \mathrm{~Hz}$. Four values were averaged to give a final sampling frequency of $128 \mathrm{~Hz}$, well above the Nyquist frequency. The resulting electrical power spectra were divided into 6 specially defined frequency ranges (delta: $0.8 \mathrm{~Hz}-4.5 \mathrm{~Hz}$; theta: $4.75 \mathrm{~Hz}-6.75 \mathrm{~Hz}$; alpha1: $7.00 \mathrm{~Hz}-9.50 \mathrm{~Hz}$; alpha2: $9.75 \mathrm{~Hz}-12.50 \mathrm{~Hz}$; beta1: $12.75 \mathrm{~Hz}-18.50 \mathrm{~Hz}$; beta2: $18.75 \mathrm{~Hz}-35.00 \mathrm{~Hz}$ ). Spectra were averaged in steps of 3 minutes each and displayed on-line. In an off-line procedure spectra were averaged to give longer periods for further analysis and data presentation.

In a preliminary study we established the highest dose to be applied as $50 \mathrm{mg} / \mathrm{kg}$ body weight (not shown). 
Three doses of OE, diluted in corn oil (Dyets, Inc., Bethlehem, PA, USA), were tested in this study by oral administration. The "Tele-Stereo-EEG" animal model consisting of continuous recording of intracerebral field potentials was used in combination with a video tracking system for detection of changes in motility (GJB Datentechnik GmbH, 98704 Langewiesen, Germany). This system, first validated in a study with different doses of caffeine in rats, recognizes locomotion as well as stereotyped behavior by following a contrast difference of the black transmitter on the head of the animal in comparison to its surrounding. After a period $45 \mathrm{~min}$ of baseline recording, freshly prepared OE dilutions were administered orally with a continuation of recordings for an additional 305 minutes.

\subsection{Surgery}

Rats were implanted with 4 bipolar concentric steel electrodes within a stereotactic surgical procedure during anesthesia with ketamine. All four electrodes were placed 3 $\mathrm{mm}$ lateral within the left hemisphere. Dorso-ventral coordinates were 4, 6, 4.2 and $8 \mathrm{~mm}$ and anterior coordinates were 3.7, 9.7, 5.7 and $12.2 \mathrm{~mm}$ for frontal cortex, striatum, hippocampus, and reticular formation, respectively (according to the rat brain atlas [26]). A pre-constructed base plate carrying 4 bipolar stainless steel semi-micro electrodes (neurological electrodes "SNF 100" from Rhodes Medical Instruments Inc., Summerland, CA 93067, USA) and a 5-pin-plug was fixed to the skull by dental cement interacting with 3 steel screws placed on distance into the bone. The distant recording spot of the electrode was the active electrode whereas the proximal spots of the four electrodes were connected to each other to give a reference as previously published [20]. The base plate was carrying a plug to receive the transmitter later during the experiment (weight: $5.2 \mathrm{~g}$ including battery, $26 \times 12 \times 6 \mathrm{~mm}$ of size). Two weeks after surgery, implanted animals were exposed to three $\mathrm{OE}$ doses in a crossover design receiving one dose each week. Animals were dosed orally $(12.5,25$ and $50 \mathrm{mg} / \mathrm{kg}$ body weight) at a constant volume of $1 \mathrm{ml}$ solution per $\mathrm{kg}$ of body weight. Control-treatment consisted of oral administration of vehicle, i.e. $1 \mathrm{ml} / \mathrm{kg}$ of corn oil solution $(\mathrm{n}=8)$. After a base-line recording lasting 45 minutes, the test compounds were administered followed by a break of $5 \mathrm{~min}$ before restart of the recording for 5 hours subdivided into 15 min periods. Changes of electrical power $\left(\mu \mathrm{V}^{2} / \Omega\right)$ are expressed as \% of the absolute base-line spectral power values within each frequency band. Field potential changes were used to construct an electropharmacogram over four standardized brain areas for each animal. Frequency spectra were obtained by Fast Fourier Transformation (FFT) and results were averaged representing 15 min periods.

\subsection{Discriminant Analysis of Electropharmacograms}

A quantitative comparison of 24 variables (four brain areas combined with six frequency ranges) was done using the mathematical procedure of discriminant analysis as described earlier. Discriminant analysis allows a search for quantitative similarities between the electropharmacograms of the test compounds and reference drugs with known clinical indication.

\subsection{Statistics}

Statistics were calculated according to the Wilcoxon, Mann Whitney U-test and by discriminant analysis. In this mathematical procedure drugs with similar clinical indication group together in space, according to the results of the first three discriminant functions, which are projected into $\mathrm{x}, \mathrm{y}, \mathrm{z}$ space. For identification of these reference drugs please consult Table 1. Major differences between drug actions are explained by the result of these first three discriminant functions. The results of the next three discriminant functions are coded into color. An additive color mixture of red (fourth function), green (fifth function) and blue (sixth function) is used to document more slight differences between actions of drugs. This means that close neighborhood and similar color indicate a very close similarity of action. Data were averaged from each group and were expressed as mean values \pm S.E.M.

\section{Results}

\subsection{Oral OE Administration Alters Electropharmacogram in Brains of Freely Moving Rats}

The electropharmacograms were recorded continuously for $5 \mathrm{~h}$ after the administration of vehicle or different doses of OE. No changes of spectral power within the four brain regions were observed following the administration of the vehicle, corn oil (not shown). Spectral frequency changes in the presence of the lowest dosage of OE (12.5 $\mathrm{mg} / \mathrm{kg}$ ) were predominantly seen in the frontal cortex. Statistically significant decreases of electrical power could be documented for delta, theta and alpha waves. Some significant, but less robust, decreases in alpha wave spectral powers were also observed within the reticular formation (Figure 1). The effects only lasted for the first hour after administration. Doubling OE to an intermediate dose of $25 \mathrm{mg} / \mathrm{kg}$ resulted in identical, but more robust, spectral frequency changes in the frontal cortex. In addition, statistically significant decreases were also observed with respect to beta waves in frontal cortex and alpha2 waves in midbrain reticular formation and striatum. Moreover, a trend towards a decrease of alpha2 electrical power was 
Table 1. Listing of reference compounds used for discriminant analysis (see Figure 5). Doses and time of recording are given. Most compounds are administered intraperitoneally except for sedative compounds and OE, which are given orally. Please note that anticonvulsive drugs need longest time to affect the encephalograms. Drugs are colored according to clinical indications: analgesics (pink), stimulants (brown), anorectics (yellow) and antidpressive/neuroprotective (green), neuroleptics (light blue), sedatives (dark blue), anticonvulsive and hallucinogenics (violet).

\begin{tabular}{|c|c|c|c|}
\hline NO. & Substance & Dose $[\mathrm{mg} / \mathrm{kg}]$ & Time \\
\hline ASS & Acetylsaliycl acid & 200 & $5-35 \min$ \\
\hline MTD & Methadon & 1 & $5-35 \min$ \\
\hline MET & Metamizol & 100 & $5-35 \mathrm{~min}$ \\
\hline FEN & Fentanyl & 0.075 & $5-35 \min$ \\
\hline MNT & Metanicotine & 1 & $5-35 \min$ \\
\hline $\mathrm{CAF}$ & Caffeine & 1 & $5-35 \mathrm{~min}$ \\
\hline AMP & (+) Amphetamine & 0.2 & $5-35 \min$ \\
\hline FEN & Fenfluramine & 1 & $5-35 \min$ \\
\hline RIM & Rimonabant & 2 & $5-35 \mathrm{~min}$ \\
\hline MPD & Methylphenidat & 2.5 & $5-35 \min$ \\
\hline SEL & Selegiline & 1.5 & $5-35 \mathrm{~min}$ \\
\hline PRA & Pramipexole & 0.3 & $5-35 \min$ \\
\hline RAS & Rasagiline & 0.5 & $5-35 \mathrm{~min}$ \\
\hline TAC & Tacrine & 0.75 & $5-35 \min$ \\
\hline GAL & Galantamine & 1 & $5-35 \mathrm{~min}$ \\
\hline DON & Donepezil & 1.5 & $5-35 \mathrm{~min}$ \\
\hline PAR & Paroxetine & 2 & $5-35 \mathrm{~min}$ \\
\hline MEM & Memantine & 3 & $5-35 \mathrm{~min}$ \\
\hline AMI & Amitryptiline & 10 & $5-35 \min$ \\
\hline MIA & Mianserine & 5 & M \\
\hline IMI & Imipramine & 10 & $5-35 \mathrm{~min}$ \\
\hline FLU & Fluvoxamine & 40 & $5-35 \mathrm{~min}$ \\
\hline CHL & Chlorpromazine & 0.5 & $5-35 \min$ \\
\hline HAL & Haloperidol & 0.5 & $5-35 \min$ \\
\hline THI & Thioridazine & 5 & $5-35 \mathrm{~min}$ \\
\hline CLO & Clozapine & 3 & $5-35 \min$ \\
\hline REM & Olanzapine & 5 & $5-35 \min$ \\
\hline OLA & Quetiapine & 6 & $5-35 \mathrm{~min}$ \\
\hline QUE & Quetiapine & 2.5 & $5-35 \min$ \\
\hline RIS & Risperidone & 0.25 & $5-35 \mathrm{~min}$ \\
\hline ZIP & Ziprasidone & 2.5 & $5-35 \min$ \\
\hline MHT & Methohexital & 20 & $20-50 \mathrm{~min}$ \\
\hline MEP & Meprobamate & 60 & $20-50 \mathrm{~min}$ \\
\hline MID & Midazolam & 0.5 & $20-50 \mathrm{~min}$ \\
\hline DIA & Diazepam & 0.5 & $20-50 \mathrm{~min}$ \\
\hline PHE & Phenobarbitone & 60 & $20-50 \mathrm{~min}$ \\
\hline VAL & Valproic acid & 75 & $65-125 \min$ \\
\hline PTO & Phenytion & 4 & $65-125 \min$ \\
\hline CAR & Carbamazepine & 15 & $65-125 \min$ \\
\hline GAB & Gabapentine & 30 & $65-125 \mathrm{~min}$ \\
\hline LSD & LSD & 0.05 & $5-35 \min$ \\
\hline DOM & R-DOM & 0.2 & $5-35 \min$ \\
\hline DIZ & Dizocilpine & 0.25 & $5-35 \min$ \\
\hline
\end{tabular}


seen in the hippocampus with an error probability of $10 \%$ $(p<0.10)$. These effects were also of short duration, not lasting more than one hour after OE administration (Fi- gure 2), suggesting a transient effect of OE on brain neurotransmission.

Administration of the highest dosage of $50 \mathrm{mg} / \mathrm{kg}$ led

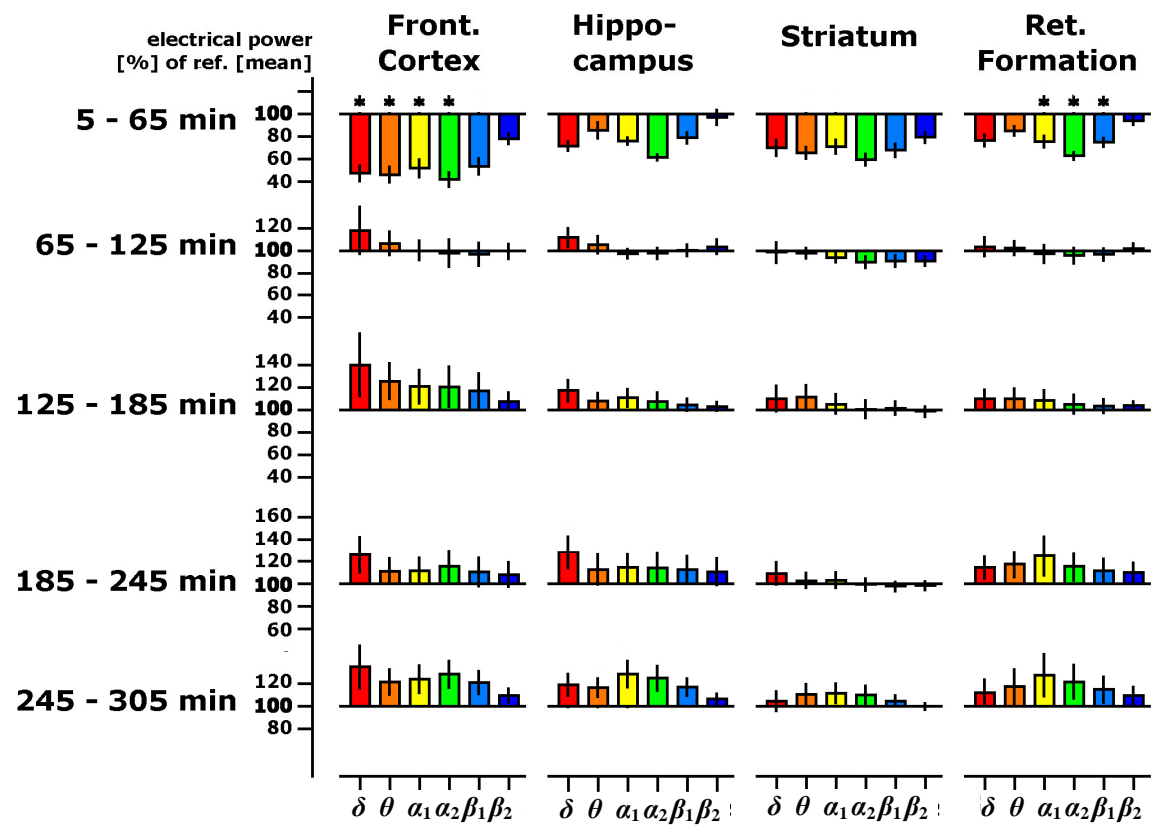

Figure 1. Time-dependent changes of spectral power presented as \% of the $45 \mathrm{~min}$ lasting pre-drug baseline recording in four brain regions of the freely moving rats (OE dose: $12.5 \mathrm{mg} / \mathrm{kg}$ body weight). Frequency ranges are depicted as colored bar graphs representing delta, theta, alpha1, alpha2, beta1 and beta2 spectral power from left to right within the four brain areas as mentioned on top of the graph. Data were averaged to give mean values in hourly intervals. *: p < 0.05: significantly different from the control (vehicle) treatment (corn oil).

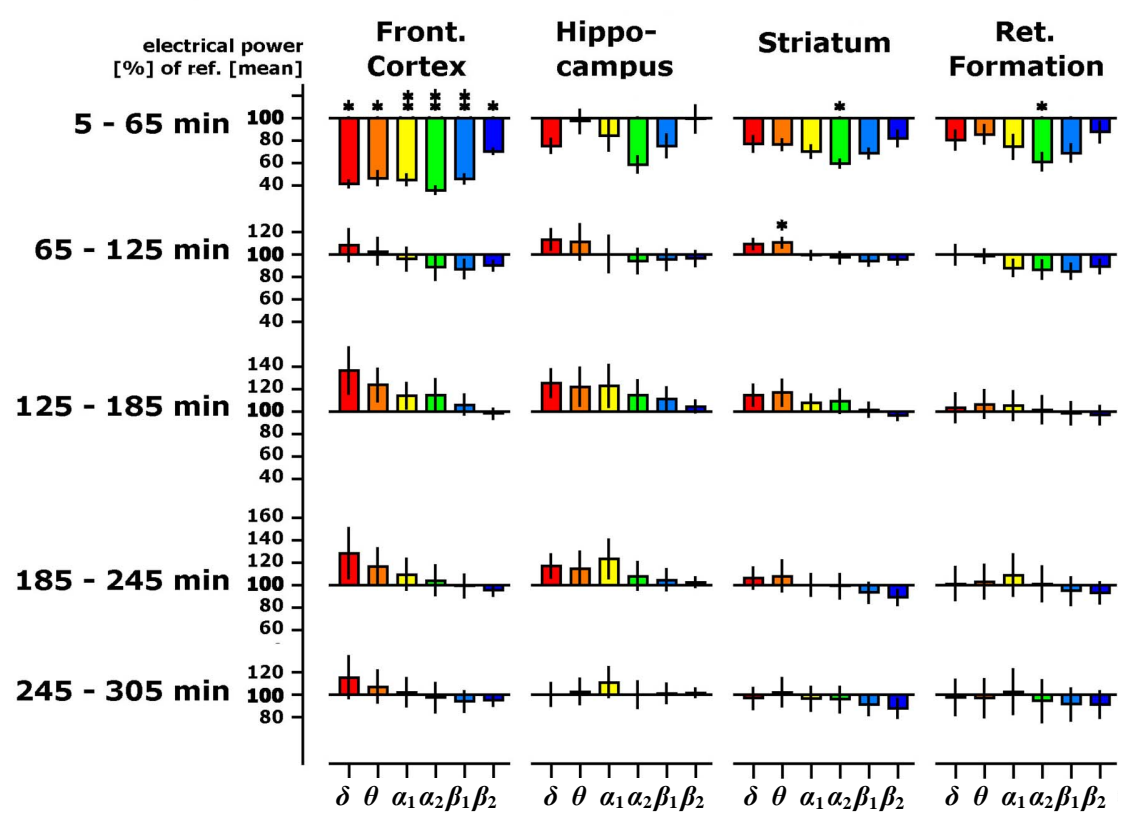

Figure 2. Time-dependent changes of spectral delta, theta, alpha1, alpha2, beta1 and beta2 spectral powers presented as \% of the $45 \mathrm{~min}$ lasting pre-drug baseline recording in four brain regions of the freely moving rats (OE dose: $25 \mathrm{mg} / \mathrm{kg}$ body weight). Data were averaged to give mean values in hourly intervals. ${ }^{*}: \mathbf{p}<0.05, * *$ : $p<0.01$ : significantly different from the vehicle treatment (corn oil). 
to same pattern of spectral frequency changes as observed for the lower dosages. Highly significant decreases of electrical power were seen in the frontal cortex with respect to delta, theta, alpha and also beta frequencies (Figure 3). Smaller, but still significant changes in comparison to control could also be seen in all brain areas studied, with the alpha1, alpha 2 and beta1 waves exhibiting the most prominent decreases of power. Oral administration of the highest dose resulted in changes that also could be observed during the $2^{\text {nd }}$ hour after administration but no further changes could be observed after this time (Figure 3). Taken together these data show a temporary, time and dose-dependency of OE intake on spectral brain activities.

A careful analysis of data revealed that changes in the spectral power of alpha and beta waves in the frontal cortex and reticular formation as soon as 5 - 20 minutes after administration of the lowest OE dose (Figure 4), further providing the evidence that $\mathrm{OE}$ is brain active and the effects on the brain function can start as quickly as 5 minutes after oral administration.

Concomitantly with the telemetric recordings, motion was monitored throughout the whole recording period. There was a tendency of higher motility in the presence of the middle dosage, which did not reach statistical significance (not shown).

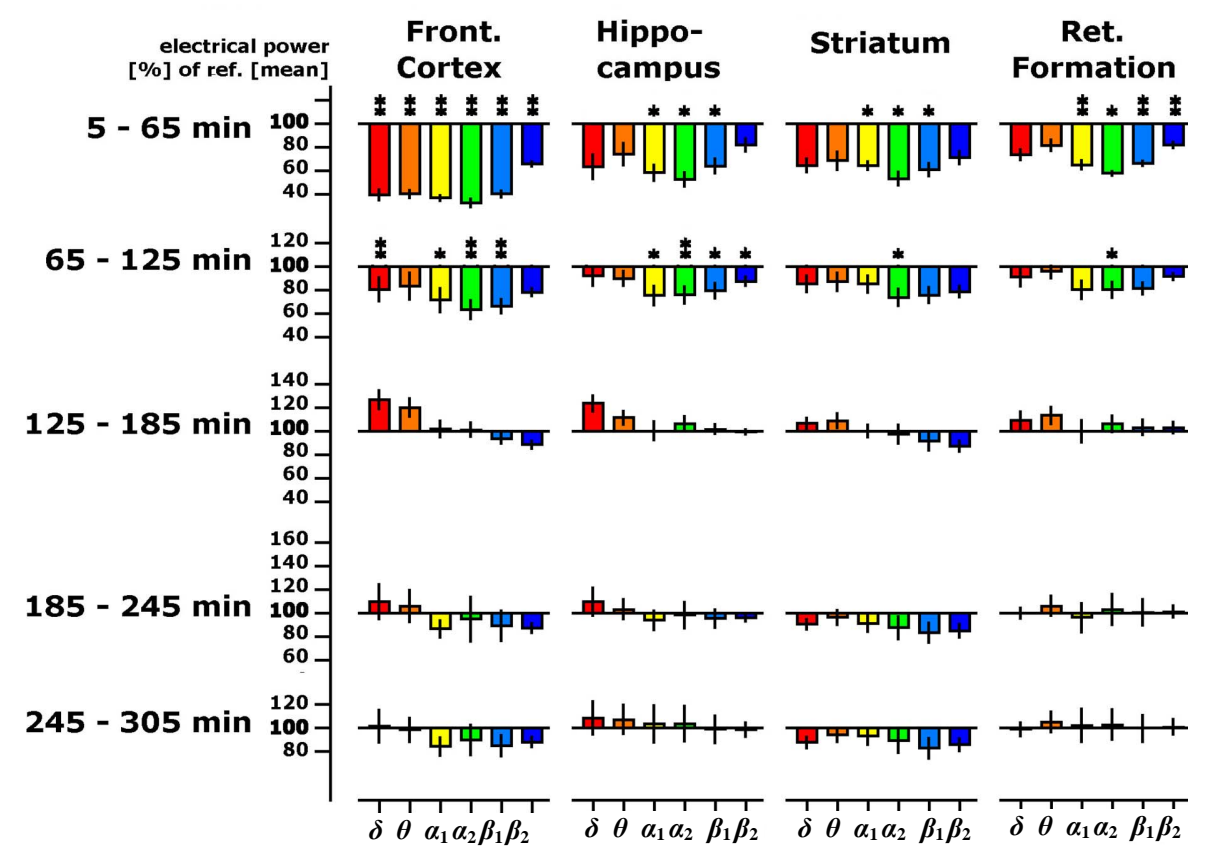

Figure 3. Effects of OE supplementation $(50 \mathrm{mg} / \mathrm{kg}$ body weight) on delta, theta, alpha1, alpha2, beta1 and beta2 spectral powers as described for Figure $1 . *: \mathbf{p}<0.05, * *: p<0.01$ : significantly different from the vehicle (corn oil) treatment.

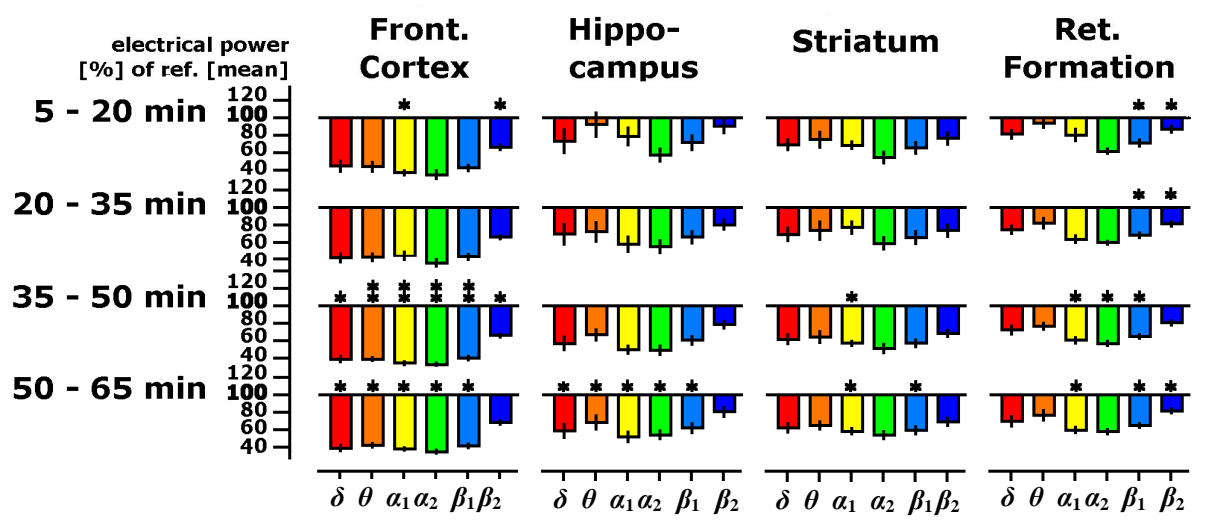

Figure 4. The changes of delta, theta, alpha1, alpha2, beta1 and beta2 spectral powers in the first hour after oral OE supplementation (50 mg/kg body weight) provide the evidence that $\mathrm{OE}$ affects brain electrical activity quickly after oral supplementation. $*$ : $\mathbf{p}<0.05, * *$ : $\mathbf{p}<0.01$ : significantly different from the vehicle treatment (corn oil). 


\subsection{Discriminant Analysis of Electropharmacograms}

We used the discriminant analysis to compare the pattern of frequency changes generated by $\mathrm{OE}$ with pharmacological effects of known compounds [20-22]. Spherical projection of the result of this calculation led to an obvious separation of the action of OE. Using the three spatial coordinates for the first three discriminant axes and green, red and blue color for the depiction of the result of the second three discriminant axes, indication-specific activity changes can be visualized by grouping of reference compounds. Figure 5 documents this indication specific grouping, which correspond quite well to pharmacological text books for the effects of tested drugs and clinical indications (see listing in Table 1). Firstly, the three-dimensional space gives evidence of similarity to neighboring drug actions. In addition, equal or similar colors suggest equal or similar mechanism ring the first half hour after administration. The orally administered $\mathrm{OE}$ doses were also analyzed for the time period $20-50$ minutes after administration. Discriminant analysis reveals that all $\mathrm{OE}$ doses are labeled red, indicating the same biological activities of all three dose. Additional, all three doses, localize near the antidepressive and neuroprotective compound such as moclobemide (MOC, a MAO A inhibitor and paroxetine (PAR, a selective ser- tonin reuptake inhibiter; SSRI) (Figure 5).

\section{Discussion}

The results of this study show that a specific supercritical- $\mathrm{CO}_{2}$ extract of oregano leaves (oreVida ${ }^{\mathrm{TM}}$, a proprietary product of DSM Nutritional Products) has neuroprotective and mood-improving capacities in vivo based on similar patterns of spectral frequencies observed by reference drugs of these clinical indications. These effects were time and dose dependent as has already been shown for other natural compounds like rutin and quercetin [22].

The changes of spectral frequency over time varied slightly depending on the applied OE dose. In addition to reaching putative active concentrations in the brain by administration of higher doses, dose-dependent differences might also arise from the varying adsorption kinetics in the stomach and the gut. Nevertheless, all applications produced very similar changes in electropharmacograms. Spectral analysis revealed that alpha1 and alpha2 frequencies showed fastest and most significant spectral changes in most brain areas studied. Robust decreases of alphal waves were observed reflecting an activation of serotonergic transmission as evident by earlier trials using several drugs interacting with the serotonergic system [27]. Moreover, dopaminergic receptor antagonists produce increases of alpha2 waves in this model $[3,16]$ sug-

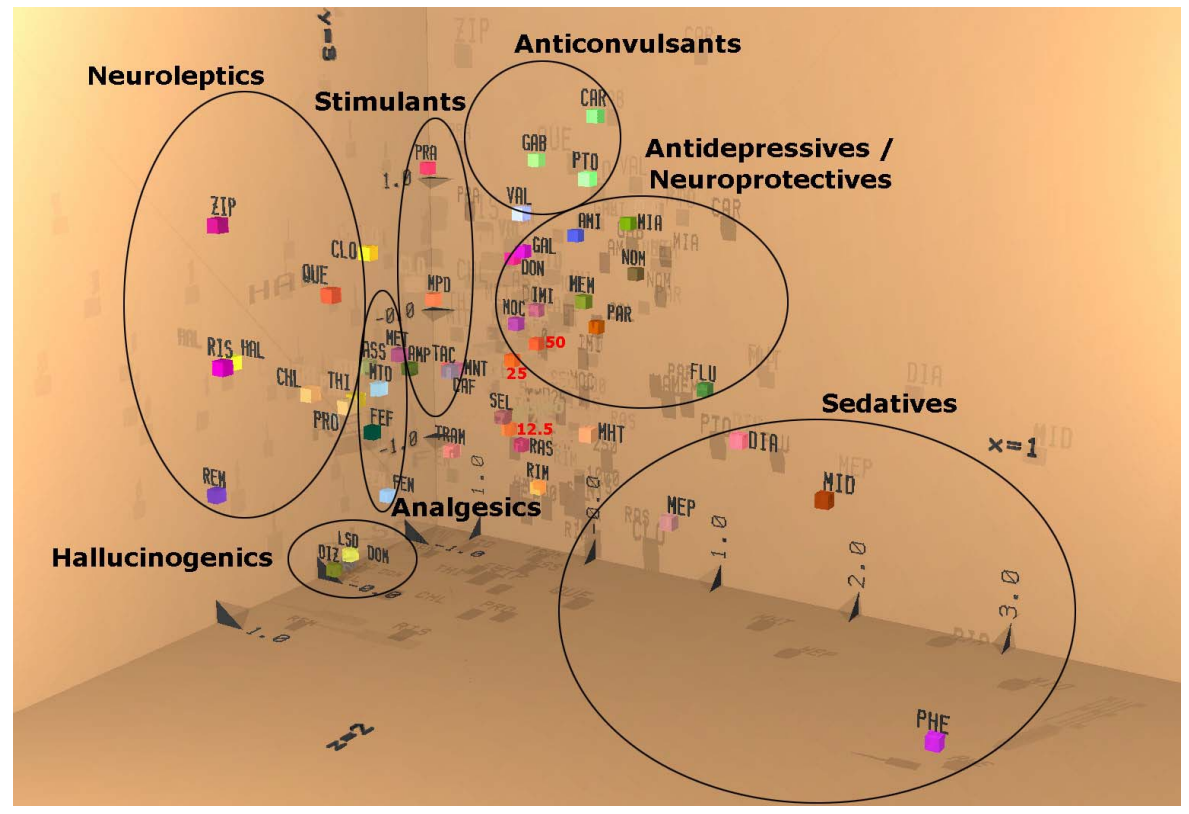

Figure 5. Discriminant analysis of electropharmacograms. Comparison of reference drugs for various clinical indications, providing similar pattern of spectral frequency changes. Compounds having a similar effect appear in close neighborhood (see $x, y$ and $z$ coordinates). Preparations with the same or similar color, due to coding of the next three discriminant axes into red, green and blue followed by an additive color mixture, have a similar mechanism of action. A great similarity with respect to space and color signalizes similar net effects with respect to clinical indication. OE (indicated as 12.5, 25, 50 for doses of $12.5 \mathrm{mg} / \mathrm{kg}, 25 \mathrm{mg} / \mathrm{kg}$, and $50 \mathrm{mg} / \mathrm{kg}$, respectively) appear in close neighborhood and with the same color. Next reference drugs are Moclobemid (MOC, a MAO A inhibitor) and Paroxetine (PAR). 
gesting that $\mathrm{OE}$ intake led to an activation of the dopaminergic system. Thus, in terms of neurotransmitter involvement, serotonergic and dopaminergic effects might be predominant after OE administration. These results are in agreement with our previous data that $\mathrm{OE}$, but none if its constituents, acts as a moderate natural triple-reuptake inhibitor reducing the cellular uptake of serotonin, dopamine and noradrenalin in vitro and increases dose-dependently brain serotonin levels in vivo [4]. In addition, especially after administration of the highest dose, we also observed decreases in delta, theta and betal waves. Changes in delta waves in the presence of pharmacological interference have been associated with the cholinergic system; i.e. decreases of delta waves are linked with an activation of the cholinergic system [13]. Accordantly, decreases of theta waves could be related to interference with the noradrenalin alpha2 receptor [14] and betal reductions can be interpreted as an activation of the glutamatergic transmission [16]. Therefore, $\mathrm{OE}$ led to a general activation of glutamatergic transmission as previously shown for a variety of antidepressive drugs in our model [20]. More evidence for an antidepressant-like, mood-improving effect of $\mathrm{OE}$ is also provided by discriminant analysis. Discriminant analysis compares the electrical brain activity of four distinct brain regions with those of several reference drugs, representing a whole variety of clinical indications. When OE effects at high dose were analyzed by discriminant analysis, the closest obvious similarity was to the antidepressive, mood-improving and neuroprotectives compounds. Most similar reference drugs were moclobemide, a MAO A inhibitor marketed for treatment of depression, and paroxetine, a prescribed SSRI, which is also marketed for treatment of depression. These data are in great agreement to the modeof-action of oregano extract [4]. Also in concert with previous data generated in mice, OE was tolerated well and we did not observe any side effects associated with the OE administration in this study.

Our data provide the evidence that the OE particularly activated the dopaminergic and serotonergic systems in freely moving animals in vivo, providing support that oral OE administration can be regarded as an activator of both dopaminergic and serotonergic system. These data are in concert with our data, demonstrating that $\mathrm{OE}$ inhibits moderately serotonin and dopamine re-uptake and inhibits MAO-A in vitro [4].

Similar dose and time dependent effects have been observed for all OE doses studied indicating the specificity of observed effects. Reduced serotonin and dopamine levels in the brain are associated with mood disturbances which are typically manifested as reduced motivation and increased anxiety [3]. Conversely, motivation and anxiety are often affected in mood and motivational disorders
[3,28-31]. These data and the finding that dopamine levels increase in ventral striatum during a response to reward provide the evidence for dopamine's importance for motivational behavior in humans [32,33]. In addition to antidepressant-like, mood-improving capacity, OE may also be envisaged to be helpful for increasing dopamine in people at risk for low dopamine levels, since main effects were observed with respect to the alpha2 waves representing dopaminergic activity. The fact that the low dose effects of OE map in the neighborhood of selegeline and rasagiline, two antiparkinsonian drugs [34], corroborates the anti-parkinsonian efficacy of OE.

Our data show that serotonergic and dopaminergic transmitter systems are activated transiently, but as fast as 5 20 minutes after $\mathrm{OE}$ ingestion, suggesting that $\mathrm{OE}$ reaches the brain quickly and exhibits its effects only for a limited time period. Transient and fast activation of neurotransmission combined with the fact that we did not observe any side effect associated with OE administration, suggest that the dietary use of OE will lead to a superior capability to better cope with psychological and emotional stress, and will improve motivation mood and mental wellbeing. Further trials are needed and are being planed to confirm the usefulness of dietary OE in at-risk and healthy individuals.

\section{Acknowledgements}

We greatly appreciate the experimental work as well as the data documentation performed by L. Schombert for EEG measurements. We also acknowledge the constructive input of K. Prudence during the preparation of this manuscript. oréVida ${ }^{\mathrm{TM}}$ is a trademark of DSM Nutritional Products. No conflicts of interest exist in relation to the experimental design and results described in this paper.

\section{REFERENCES}

[1] B. S. McEwen, "Physiology and Neurobiology of Stress and Adaptation: Central Role of the Brain," Physiological Reviews, Vol. 87, No. 3, 2007, pp. 873-904. doi: 10.1152/physrev.00041.2006

[2] R. Cools, K. Nakamura and N. D. Daw, "Serotonin and Dopamine: Unifying Affective, Activational, and Decision Functions," Neuropsychopharmacology, Vol. 36, No. 1, 2011, pp. 98-113. doi:10.1038/npp.2010.121

[3] W. C. Drevets, J. L. Price and M. L. Furey, "Brain Structural and Functional Abnormalities in Mood Disorders: Implications for Neurocircuitry Models of Depression," Brain Structure \& Function, Vol. 213, No. 1-2, 2008, pp 93-118. doi:10.1007/s00429-008-0189-x

[4] A. O. Mechan, A. Fowler, N. Seifert, H. Rieger, T. Wohrle, S. Etheve, A. Wyss, G. Schuler, B. Colletto, C. Kilpert, J. Aston, J. M. Elliott, R. Goralczyk and M. H. 
Mohajeri, "Monoamine Reuptake Inhibition and MoodEnhancing Potential of a Specified Oregano Extract," British Journal of Nutrition, Vol. 105, No. 8, 2011, pp. 11501163. doi: $10.1017 / \mathrm{S} 0007114510004940$

[5] K. Blum, T. J. Chen, S. Morse, J. Giordano, A. L. Chen, J. Thompson, C. Allen, A. Smolen, J. Lubar, E. Stice, B. W. Downs, R. L. Waite, M. A. Madigan, M. Kerner, F. Fornari and E. R. Braverman, "Overcoming qEEG Abnormalities and Reward Gene Deficits during Protracted Abstinence in Male Psychostimulant and Polydrug Abusers Utilizing Putative Dopamine D Agonist Therapy: Part 2," Postgraduate Medicine, Vol. 122, No. 6, 2010, pp. 214226. doi:10.3810/pgm.2010.11.2237

[6] M. J. Jutras and E. A. Buffalo, "Synchronous Neural Activity and Memory Formation," Current Opinion in Neurobiology, Vol. 20, No. 2, 2010, pp. 150-155. doi:10.1016/j.conb.2010.02.006

[7] K. Kitaoka, A. Hattori, S. Chikahisa, K. Miyamoto, Y. Nakaya and H. Sei, "Vitamin A Deficiency Induces a Decrease in EEG Delta Power during Sleep in Mice," Brain Research, Vol. 1150, 2007, pp. 121-130. doi:10.1016/j.brainres.2007.02.077

[8] M. Moazami-Goudarzi, J. Sarnthein, L. Michels, R. Moukhtieva and J. Jeanmonod, "Enhanced Frontal Low and High Frequency Power and Synchronization in the Resting EEG of Parkinsonian Patients," Neuroimage, Vol. 41, No. 3, 2008, pp. 985-997.

doi:10.1016/j.neuroimage.2008.03.032

[9] W. Dimpfel, A. Kler, E. Kriesl, R. Lehnfeld and I. K. Keplinger-Dimpfel, "Source Density Analysis of the Human EEG after Ingestion of a Drink Containing Decaffeinated Extract of Green Tea Enriched with L-Theanine and Theogallin," Nutritional Neuroscience, Vol. 10, No. 3-4, 2007, pp.169-180. doi:10.1080/03093640701580610

[10] W. Dimpfel, "Preclinical Data Base of Pharmaco-Specific Rat EEG Fingerprints (Tele-Stereo-EEG)," European Journal of Medical Research, Vol. 8, No. 5, 2003, pp. 199207.

[11] A. Czubak, E. Nowakowska, K. Burda, K. Kus and J. Metelska, "Cognitive Effects of GABAergic Antiepileptic Drugs," Arzneimittelforschung, Vol. 60, No. 1, 2010, pp. 1-11. doi:10.1055/s-0031-1296242

[12] A. G. Malykh and M. R. Sadaie, "Piracetam and Piracetam-Like Drugs: From Basic Science to Novel Clinical Applications to CNS Disorders," Drugs, Vol. 70, No. 3, 2010, pp. 287-312. doi:10.2165/11319230-000000000-00000

[13] W. Dimpfel, "Pharmacological Modulation of Cholinergic Brain Activity and Its Reflection in Special EEG Frequency Ranges from Various Brain Areas in the Freely Moving Rat (Tele-Stereo-EEG)," European Neuropsychopharmacology, Vol. 15, No. 6, 2005, pp. 673-682. doi:10.1016/j.euroneuro.2005.03.006

[14] W. Dimpfel and F. Schober, "Norepinephrine, EEG Theta Waves and Sedation," Pharmacology, Vol. 1, 2001, pp. 89-97.

[15] W. Dimpfel, "Pharmacological Modulation of Dopaminergic Brain Activity and Its Reflection in Spectral Fre- quencies of the Rat Electropharmacogram," Neuropsychobiology, Vol. 58, No. 3-4, 2008, pp. 178-186. doi:10.1159/000191124

[16] W. Dimpfel, M. Spuler, R. Koch and W. Schatton, "Radioelectroencephalographic Comparison of Memantine with Receptor-Specific Drugs Acting on Dopaminergic Transmission in Freely Moving Rats," Neuropsychobiology, Vol. 18, No. 18, 1987, pp. 212-218. doi: $10.1159 / 000118420$

[17] S. Ortigue, N. Patel and F. Bianchi-Demicheli, "New Electroencephalogram (EEG) Neuroimaging Methods of Analyzing Brain Activity Applicable to the Study of $\mathrm{Hu}-$ man Sexual Response," The Journal of Sexual Medicine, Vol. 6, No. 7, 2009, pp. 1830-1845. doi:10.1111/j.1743-6109.2009.01271.x

[18] F. Pulvermuller, Y. Shtyrov and O. Hauk, "Understanding in an Instant: Neurophysiological Evidence for Mechanistic Language Circuits in the Brain," Brain and Language, Vol. 110, No. 2, 2009, pp. 81-94. doi:10.1016/j.bandl.2008.12.001

[19] J. M. Schoffelen and J. Gross, "Source Connectivity Analysis with MEG and EEG," Human Brain Mapping, Vol. 30, No. 6, 2009, pp.1857-1865. doi:10.1002/hbm.20745

[20] W. Dimpfel, M. Spuler and H. O. Borbe, "Monitoring of the Effects of Antidepressant Drugs in the Freely Moving Rat by Radioelectroencephalography (Tele-Stereo-EEG)," Neuropsychobiology, Vol. 19, No. 2, 1988, pp. 116-120. doi: $10.1159 / 000118445$

[21] G. S. Ruigt, S. Engelen, A. Gerrits and F. Verbon, "Computer-Based Prediction of Psychotropic Drug Classes Based on a Discriminant Analysis of Drug Effects on Rat Sleep," Neuropsychobiology, Vol. 28, No. 3, 1993, pp. 138-153. doi:10.1159/000119016

[22] W. Dimpfel, "Rat Electropharmacograms of the Flavonoids Rutin and Quercetin in Comparison to Those of Moclobemide and Clinically Used Reference Drugs Suggest Antidepressive and/or Neuroprotective Action," Phytomedicine, Vol. 16, No. 4, 2009, pp. 287-294. doi:10.1016/j.phymed.2009.02.005

[23] W. Dimpfel, M. Spuler and H. G. Menge, "Effects of the Antiparkinson Drug Budipine on EEG Activity in Unrestrained Rats," Arzneimittelforschung, Vol. 39, No. 5, 1989, pp. 560-563.

[24] W. Dimpfel, M. Spuler and D. E. Nichols, "Hallucinogenic and Stimulatory Amphetamine Derivatives: Fingerprinting DOM, DOI, DOB, MDMA, and MBDB by Spectral Analysis of Brain Field Potentials in the Freely moving Rat (Tele-Stereo-EEG)," Psychopharmacology (Berl), Vol. 98, No. 3, 1989, pp. 297-303. doi:10.1007/BF00451678

[25] W. Dimpfel, M. Spuler and B. Nickel, "Radioelectroencephalography (Tele-Stereo-EEG) in the Rat as a Pharmacological Model to Differentiate the Central Action of Flupirtine from That of Opiates, Diazepam and Phenobarbital," Neuropsychobiology, Vol. 16, No. 2-3, 1986, pp. 163-168. doi:10.1159/000118319

[26] G. Paxinos and C. Watson, "The Rat Brain in Stereotactic Coordinates,” Academic Press, New York, 1982. 
[27] W. Dimpfel, M. Spuler and B. Nickel, "Dose- and TimeDependent Action of Morphine, Tramadol and Flupirtine as Studied by Radioelectroencephalography in the Freely Behaving Rat," Neuropsychobiology, Vol. 20, No. 3, 1989, pp. 164-168. doi:10.1159/000118492

[28] J. L. Price and W. C. Drevets, "Neurocircuitry of Mood Disorders," Neuropsychopharmacology, Vol. 35, No. 1, 2010, pp. 192-216. doi:10.1038/npp.2009.104

[29] K. J. Ressler and H. S. Mayberg, "Targeting Abnormal Neural Circuits in Mood and Anxiety Disorders: From the Laboratory to the Clinic," Nature Neuroscience, Vol. 10, No. 9, 2007, pp. 1116-1124. doi:10.1038/nn1944

[30] L. M. Shin and I. Liberzon, "The Neurocircuitry of Fear, Stress, and Anxiety Disorders" Neuropsychopharmacology, Vol. 35, No. 1, 2010, pp. 169-191. doi:10.1038/npp.2009.83

[31] R. Cools, "Role of Dopamine in the Motivational and Cognitive Control of Behavior," Neuroscientist, Vol. 14, No.

\section{4, 2008, pp. 381-395. doi:10.1177/1073858408317009}

[32] M. Pessiglione, B. Seymour, G. Flandin, R. J. Dolan and C. D. Frith, "Dopamine-Dependent Prediction Errors Underpin Reward-Seeking Behaviour in Humans," Nature, Vol. 442, No. 7106, 2006, pp. 1042-1045.

doi:10.1038/nature05051

[33] B. H. Schott, L. Minuzzi, R. M. Krebs, D. Elmenhorst, M. Lang, O. H. Winz, C. I. Seidenbecher, H. H. Coenen, H. J. Heinze, K. Zilles, E. Duzel and A. Bauer, "Mesolimbic Functional Magnetic Resonance Imaging Activations during Reward Anticipation Correlate with Reward-Related Ventral Striatal Dopamine Release," Journal of Neuroscience, Vol. 28, No. 28, 2008, pp. 14311-14319. doi:10.1523/JNEUROSCI.2058-08.2008

[34] W. Dimpfel and J. A. Hoffmann, "Electropharmacograms of Rasagiline, Its Metabolite Aminoindan and Selegiline in the Freely Moving Rat," Neuropsychobiology, Vol. 62, No. 4, 2010, pp. 213-220. doi:10.1159/000319947 